\title{
Preclinical Evaluation of the Thrombogenicity and Endothelialization of Bare Metal and Surface-Coated Neurovascular Stents
}

\author{
S. Krajewski, B. Neumann, J. Kurz, N. Perle, M. Avci-Adali, G. Cattaneo, and H.P. Wendel
}

\begin{abstract}
BACKGROUND AND PURPOSE: Stent-assisted coiling is routinely used for the endovascular treatment of complex or wide-neck intracranial aneurysms. However, in-stent thrombosis, thromboembolic events, and ischemic complications remain a major concern associated with stent implants. Therefore, a novel low-profile neurovascular stent with a bare metal surface was investigated with regard to thrombogenicity and endothelialization and compared with the same stent coated with albumin or heparin.
\end{abstract}

MATERIALS AND METHODS: The bare metal and heparin- or albumin-coated stents were loaded in heparin-coated tubing, which was then filled with heparinized human blood $(n=5)$ and circulated at $150 \mathrm{~mL} / \mathrm{min}$ and $37^{\circ} \mathrm{C}$ for 60 minutes. Before and after circulation, measurement of various inflammation and coagulation markers and scanning electron microscopy were performed. Endothelialization of the stents was investigated in an in vitro model including human umbilical vascular endothelial cells.

RESULTS: Our results showed that platelet loss and platelet activation and activation of the coagulation cascade, which are induced by the bare metal stents, were significantly reduced by heparin or albumin coating. Adverse effects on erythrocytes, leukocytes, and the complement cascade were not induced by the bare metal or coated stents. Moreover, the bare metal and albumin-coated stents showed good endothelialization properties.

CONCLUSIONS: Albumin and heparin coatings clearly improve the thrombogenicity of the stents and thus may represent fundamental progress in the treatment of intracranial aneurysms. Moreover, preclinical evaluation of neurovascular stents under physiologic conditions supports and accelerates the development of more biocompatible neurovascular stents.

ABBREVIATIONS: HUVECs = human umbilical vascular endothelial cells; SEM = scanning electron microscopy; TAT = thrombin-antithrombin III

$\mathbf{E}^{\mathrm{n}}$ ndovascular treatment of complex or wide-neck intracranial - aneurysms has been proved feasible by using the stent-assisted coiling technique, which was primarily described in $1997 .{ }^{1}$ In this procedure, self-expandable, nitinol-made stents, braided or laser cut, are delivered into the parent vessel by using microcatheters with an inner lumen between 0.0165 and 0.027 inches to retain coils within the aneurysm. ${ }^{1,2}$

Thus, the implanted stent prevents coil protrusion within the

Received March 6, 2014; accepted after revision June 23.

From the Department of Thoracic, Cardiac and Vascular Surgery (S.K., B.N., J.K., N.P., M.A.-A., H.P.W.), Clinical Research Laboratory, University Hospital Tuebingen, Tuebingen, Germany; and Acandis GmbH \& Co KG (G.C.), Pforzheim, Germany.

The study was funded by the German Federal Ministry of Economic Affairs (Economics and Energy).

Please address correspondence to Stefanie Krajewski, PhD, Department of Thoracic, Cardiac and Vascular Surgery, University Hospital Tuebingen, Clinical Research Laboratory, Tuebingen University, Calwerstr 7/1, 72076 Tuebingen, Germany; e-mail: stefanie.krajewski@uni-tuebingen.de

- Indicates open access to non-subscribers at www.ajnr.org

http://dx.doi.org/10.3174/ajnr.A4109 parent vessel, reduces coil compaction and hence recanalization, and provides a scaffold for endothelialization. ${ }^{2-5}$

However, the endoluminal presence of the implant can lead to activation of platelets and leukocytes following adverse effects like thromboembolic complications, hemorrhage, or proinflammatory reactions. Platelet activation is associated with the release of granules containing a plethora of molecules mainly promoting platelet activation and blood coagulation. Therefore, antiplatelet therapy is required, which can prevent intra-aneurysmal thrombosis and thus aneurysm occlusion but also increases the risk of hemorrhagic complications. ${ }^{6}$ Moreover and despite antiplatelet therapy, ischemic complications remain a major concern associated with stent implants. A retrospective review of stent-assisted coiling procedures in 624 patients between 2000 and 2011 revealed intra- and postprocedural in-stent thrombosis and thromboembolic events in $9.0 \%$ of the patients, whereas low or no response to antiplatelet agents and early interruption of therapy are possible causes for ischemic events. ${ }^{2}$

A retrospective study of 36 patients treated with stent-assisted 
coiling after aneurysm rupture showed that in 17\%, stent-related thromboembolic complications occurred. Intra- and postprocedural clopidogrel administration was related to an incidence of thrombotic events of $20 \%$ and $29 \%$, respectively. Even pretreatment with clopidogrel could not prevent thrombotic events, which were shown in $6 \%$ of cases. $^{7}$

Therefore, preventing or strongly decreasing activation of platelets and the coagulation cascade, while enhancing or at least not impairing endothelialization, is a primary objective in the treatment of endovascular diseases. Stent design, influence on fluid dynamics, and particularly surface modifications are current options to decrease activation of platelets and the coagulation cascade, and they also address hemorrhagic stroke, because the necessity for antiplatelet therapy can be reduced. Previous studies show that surface coatings with the antithrombotic molecules heparin and albumin, which is the most abundant protein in blood, increase the biocompatibility of different biomaterials. ${ }^{8-10}$

In our study, an in vitro model was established, which allows investigation of neurovascular stents with regard to thrombogenicity and coagulation activation.

Small-diameter tubing and low flow rates simulating intracranial vessel conditions were used to investigate novel low-profile neurovascular stents with bare metal surfaces or 2 coatings, including albumin and heparin, with regard to thrombogenicity. Furthermore, a predisposition toward endothelialization was investigated in an in vitro rotation model including human umbilical vascular endothelial cells (HUVECs).

\section{MATERIALS AND METHODS}

For this study, the neurovascular stent Acclino ( $n=24$; Acandis, Pforzheim, Germany) was used. Acclino is a low-profile, laser-cut stent with an electropolished surface and strut dimensions ranging between $\sim 55 \mu \mathrm{m}$ (wall thickness) and $\sim 35$ $\mu \mathrm{m}$ (strut width).

The stent is deliverable within the 1.9F microcatheter NeuroSlider 17 (Acandis) with a lumen of 0.0165 inches $(\sim 0.42 \mathrm{~mm})$. The small catheter lumen and the size of the stent $(3.5$ and $4.5 \mathrm{~mm}$ in expanded configurations) allow the application in distal intracranial vessels with diameters between 2 and $4 \mathrm{~mm}$, whereas the closed-cell structure allows retrieval and repositioning up to $90 \%$ of its deployment.

For the current study, specific Acclino stent samples with a wall thickness of $\sim 75 \mu \mathrm{m}$ were manufactured to reproduce a worst-case scenario with regard to blood flow interruption caused by the stent structure.

\section{Blood Sampling}

Blood-sampling procedures were approved by the ethics committee of the University of Tuebingen, Tuebingen, Germany. Blood was collected from healthy volunteers $(n=5)$, who gave signed informed consent, and was anticoagulated with $1.5 \mathrm{IU} / \mathrm{mL}$ Heparin-Natrium (Rathiopharm, Ulm, Germany). Exclusion criteria for the blood donators were the following: smokers; drug intake in the last 2 weeks before blood sampling, especially hemostasisaffecting agents like acetylsalicylic acid; oral contraceptives; nonsteroidal antiphlogistics; and others, to guarantee optimal hemostatic function.

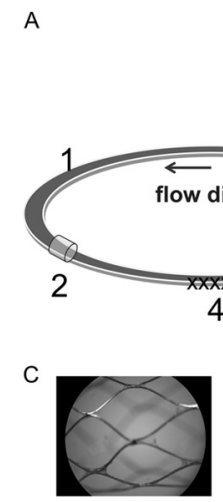

bare

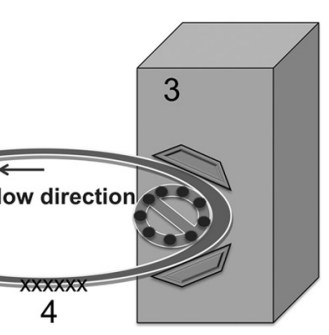

B

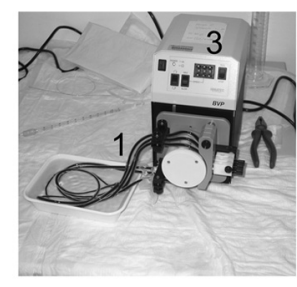

FIG 1. Closed-loop in vitro circulation model for neurovascular stent testing by using fresh human whole blood. Schematic overview $(A)$ and photograph $(B)$ of the in vitro circulation model consisting of the following: 1) heparin-coated tubing (inner diameter $=3.2 \mathrm{~mm}$, length $=75 \mathrm{~cm}$ ), 2) connection tubing, 3) peristaltic pump (flow rate = $150 \mathrm{~mL} / \mathrm{min}$ ), 4) test device. C, Representative macroscopic images of uncoated and albumin- and heparin-coated stents after circulation for 60 minutes at $37^{\circ} \mathrm{C}(\times 20$ magnification).

\section{Neurovascular Stent Coating}

For albumin coating, 8 neurovascular stents were first incubated for 2 hours at room temperature in 1\% human serum albumin (HSA; CSL Behring, Marburg, Germany), followed by a washing step in saline and a 30-minute incubation in a suspension containing 0.5 -mg of 1-ethyl-3-(3-dimethylaminopropyl)carbodiimide hydrochloride (Thermo Scientific, Karlsruhe, Germany) for albumin cross-linking. Afterward, the stents were incubated again in 1\% HSA for 2 hours at room temperature followed by another washing step in saline. After being coated, the stents were air-dried and stored at $4^{\circ} \mathrm{C}$ until analysis in the in vitro thrombogenicity model. The heparin coating of the stents $(n=8)$ for the thrombogenicity and endothelialization experiments was performed according to standard methods. ${ }^{11}$ To guarantee sterile conditions in the endothelialization model, we sterilized the stents by using ethylene oxide.

\section{In Vitro Thrombogenicity Model}

This novel model was established to investigate the effect of neurovascular stents on various cascade reactions of the human hemostatic system (coagulation cascade, cell alteration, complement system, and inflammation) during blood circulation (Fig $1 A,-B)$.

For each blood donor $(n=5), 4$ polyvinyl chloride tubes (inner diameter $=3.2 \mathrm{~mm}$, length $=75 \mathrm{~cm}$; Tygon; Saint-Gobain Performance Plastics, Chamy, France) were coated with heparin by Ension (Ension, Cape Coral, Florida) and left empty or loaded with either 1 bare metal, 1 albumin-, or 1 heparin-coated stent. Subsequently, each tubing was filled with a total of 6-mL heparinized human blood, closed by silicone connection tubing, and circulated at $150 \mathrm{~mL} / \mathrm{min}$ in a water bath at $37^{\circ} \mathrm{C}$ for 60 minutes. For each donor, $6 \mathrm{~mL}$ of heparinized blood served for the measurement of baseline values before circulation. Before and after circulation, the blood was directly transferred to the corresponding terminating media and was further processed for enzyme-linked immunosorbent assays and whole blood analysis. 

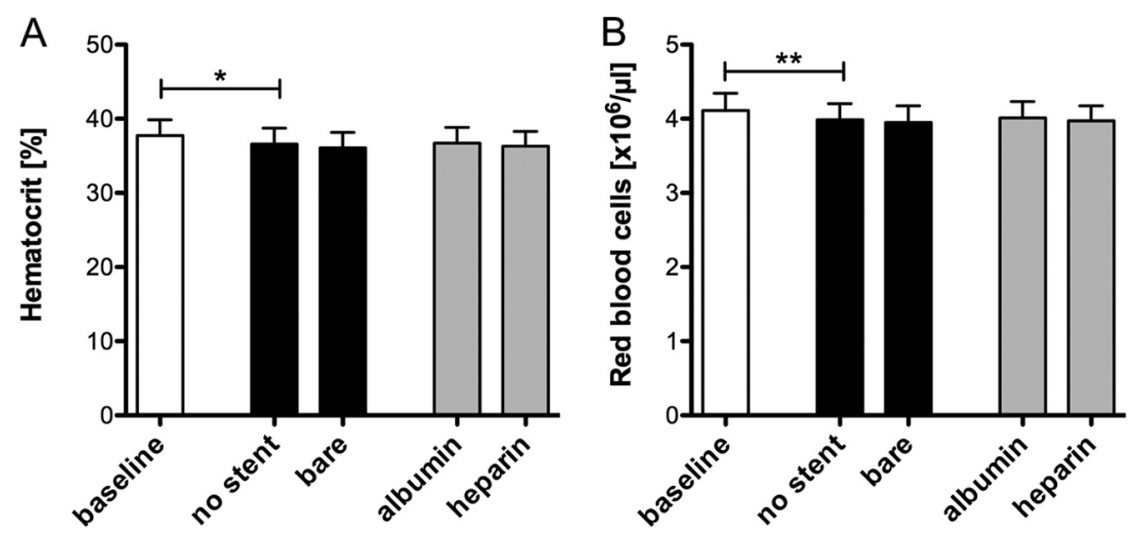

Cambridge, United Kingdom) and polymorphonuclear neutrophil elastase (Demeditec Diagnostics, Kiel-Wellsee, Germany) levels. As a marker for coagulation, we determined thrombin-antithrombin III (TAT) complexes (Siemens Healthcare, Marburg, Germany). Moreover, we analyzed SC5b-9 (TECOmedical, Bünde, Germany) to detect activation of the complement system.

\section{Whole Blood Count Analysis}

The number of erythrocytes, leukocytes, and platelets and hematocrit and hemoglobin values were measured by a cell counter system (ABX Micros 60; Axon Lab AG, Baden, Switzerland) in baseline samples and after circulation in all groups.

\section{Scanning Electron Microscopy}

Scanning electron microscopy (SEM) was performed to investigate the thrombogenicity and the endothelialization rate of the different stent surfaces after contact with human whole blood and HUVECs, respectively. After each experiment, the stents were rinsed in phosphate buffered saline and fixed in glutaraldehyde solution $(2 \%$ in phosphate buffered saline, $\mathrm{pH} 7.4$ ) overnight at $4^{\circ} \mathrm{C}$. After another washing step with phosphate buffered saline, the stents were dehydrated by using ascending concentrations of ethanol

\section{Endothelialization Model}

The endothelialization experiments $(n=3)$ were performed as previously described. ${ }^{12}$ Briefly, 2-mL bioreactor chambers were loaded with either a sterilized bare metal, an albumin-, or a heparin-coated stent. Isolation of HUVECs was adapted from a previously described method for the isolation of endothelial cells from residual saphenous vein biopsies. ${ }^{12}$ For each sample, $2.5 \times 10^{6}$ cells were resuspended in $2.5-\mathrm{mL}$ VascuLife EnGS medium (CellSystems, Troisdorf, Germany) containing a VascuLife EnGS LifeFactors Kit, 50- $\mu \mathrm{g} / \mathrm{mL}$ gentamicin, and 0.05$\mu \mathrm{g} / \mathrm{mL}$ amphotericin B (PAA Laboratories, Cölbe, Germany).

Following the addition of the cell suspension, the bioreactor chambers were assembled in the rotation device and rotated at $37^{\circ} \mathrm{C}$ and $5 \% \mathrm{CO}_{2}$. After 72 hours of incubation, the stents were washed in phosphate buffered saline and subsequently prepared for scanning electron microscopy analysis.

\section{Measurement of Hemostatic Markers}

The analysis of markers indicating activation of platelets and leukocytes and the coagulation cascade and complement system was performed in plasma by enzyme-linked immunosorbent assays, according to the manufacturer's instructions. To investigate released, plasmatic markers indicating platelet and leukocyte activation, we analyzed PF-4 (Abcam,
(Emsure; Merck, Darmstadt, Germany) and critical point dried. After sputtering with gold palladium, we performed SEM analysis by using the EVO LS 10 scanning electron microscope (Carl Zeiss, Jena, Germany).

\section{Statistics}

Data are depicted as means with standard error of the mean. Data were analyzed by using repeated-measures ANOVA with the Bonferroni multiple comparison test to analyze differences among groups. All analyses were performed by using the statistical software package GraphPad Prism (Version 5; GraphPad Software, San Diego, California). Statistical significance was defined as $P<.05$.

\section{RESULTS}

\section{Hemocompatibility Testing of Neurovascular Stents}

Contact of whole blood with artificial surfaces like stents, catheters, implants, and so forth is associated with various alterations of the hemostatic system. To evaluate the hemocompatibility of the bare metal $(n=5)$, albumin- $(n=5)$, or heparin-coated $(n=5)$ stents for neurovascular application, we developed an in vitro model to guarantee a defined and continuous blood flow mimicking blood circulation in the brain (Fig $1 A,-B$ ). Directly after 

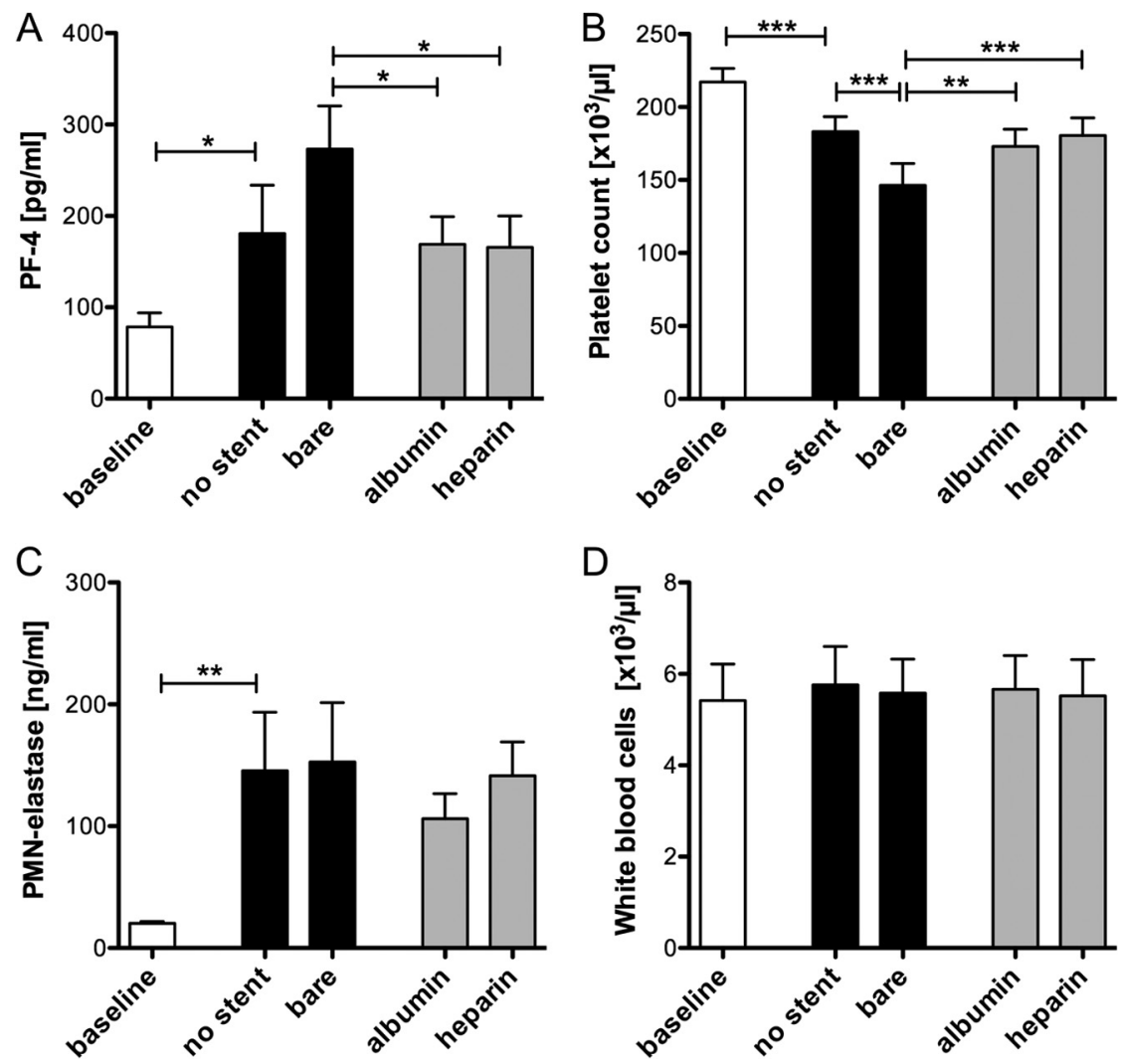

FIG 3. Platelet activation is significantly reduced due to albumin and heparin coating. Bare metal, albumin-, or heparin-coated neurovascular stents were circulated in the in vitro model for 60 minutes at $150 \mathrm{~mL} / \mathrm{min}$. Before and after circulation, $\mathrm{PF}-4$ plasma levels $(A)$, platelet counts $(B)$, polymorphonuclear neutrophil-elastase concentrations $(C)$, and white blood cell numbers (D) were analyzed in all groups. Data are given as mean and standard error of the mean $(n=5)$; groups were compared by using repeated-measures ANOVA with the Bonferroni multiple comparison test. One asterisk indicates $P<.05$; two asterisks, $P<.01$; three asterisks, $P<.001$.

A

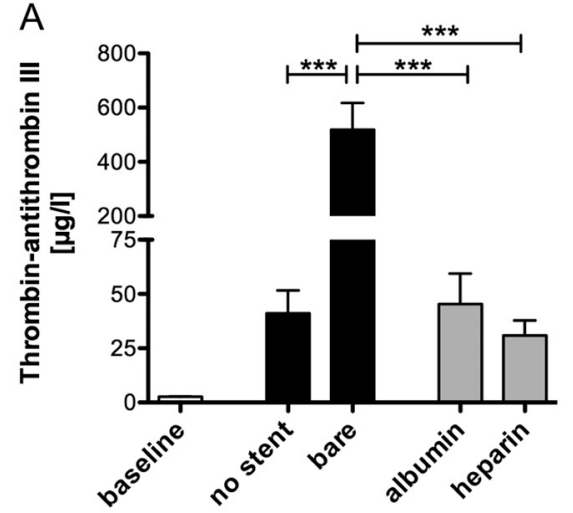

B

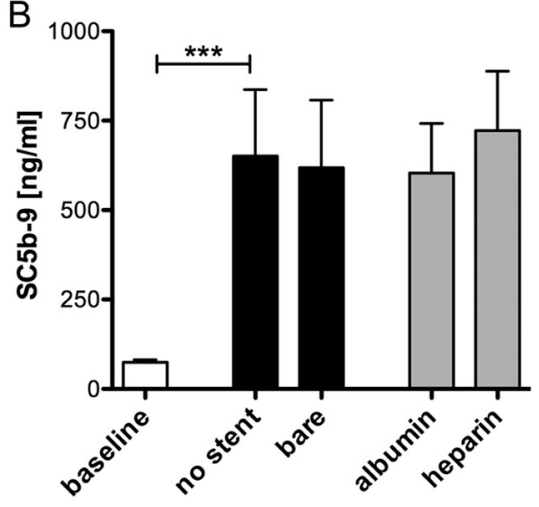

FIG 4. Albumin and heparin coating of neurovascular stents profoundly reduces activation of the coagulation cascade. TAT levels $(A)$ and Sc5b-9 concentrations $(B)$ were measured in human whole blood before and after circulation in the bare metal, albumin-, or heparin-coated stent groups. Blood circulated without a stent served as a negative control. Data are given as mean and standard error of the mean $(n=5)$, and groups were compared by using repeated-measures ANOVA with the Bonferroni multiple comparison test. Three asterisks indicate $P<.001$.

circulation, macroscopic images, which are exemplarily shown in Fig $1 C$ from 1 blood donor, were obtained of each stent.

Whole Blood Count Analysis. Before and after circulation in the in vitro model, the whole blood was analyzed in all groups. Circulation induced a significant decrease of hematocrit $(P<.05$, Fig
$2 A)$, red blood cell counts $(P<.01$, Fig $2 B)$, and hemoglobin levels $(P<.001$, Fig $2 C)$ in the control group containing no stent. No significant changes were observed in the bare metal and albumin- and heparin-coated stent groups compared with the control group.

Platelet and Leukocyte Activation. The concentration of PF-4, a small chemokine released from platelet $\alpha$ granules, is significantly increased during circulation in both control groups containing no stent and a bare metal stent, respectively $(P<$ .05 , Fig $3 A$ ). In the groups containing albumin- or heparin-coated stents, the release of PF-4 was significantly reduced $(P<.05)$. Platelet activation was also estimated by the analysis of platelet counts. Compared with a platelet count of $217.200 \pm 9.300 \mu \mathrm{L}$ before circulation, a significant loss of platelets in the control group containing no stent $(P<.001)$ was observed, which was further increased in the bare metal stent group $(P<.001$, Fig $3 B)$. Again, the decrease in platelet numbers was less severe in the coated stent groups $(P<.01)$.

Despite platelet activation, leukocyte activation based on granule release and leukocyte counts was investigated. The plasma concentration of polymorphonuclear neutrophil-elastase released from granulocytes significantly increased because of circulation in the in vitro model $(P<.01$, Fig $3 C)$. In the stent groups, no significant differences compared with the control group were detected. Similar results were obtained for the leukocyte counts (Fig 3D), in which no significant differences were detected among the groups after circulation.

Effects on the Coagulation Cascade and the Complement System. Activation of the coagulation cascade was estimated by the formation of the TAT complex indicating thrombin generation before and after circulation in all groups (Fig 4A). Compared with a TAT concentration of $41.1 \pm 10.6 \mu \mathrm{g} / \mathrm{L}$ in the group containing no stent, a significant increase in TAT concentration was found in the bare metal stent group $(518.3 \pm 98.9 \mu \mathrm{g} / \mathrm{L}, P<.001)$. Stents coated with albumin or heparin induced a significantly lower activation of the coagulation cascade $(P<.001)$, reaching values comparable with the group containing no stent.

The formation of Sc5b-9, the final complex of the complement 

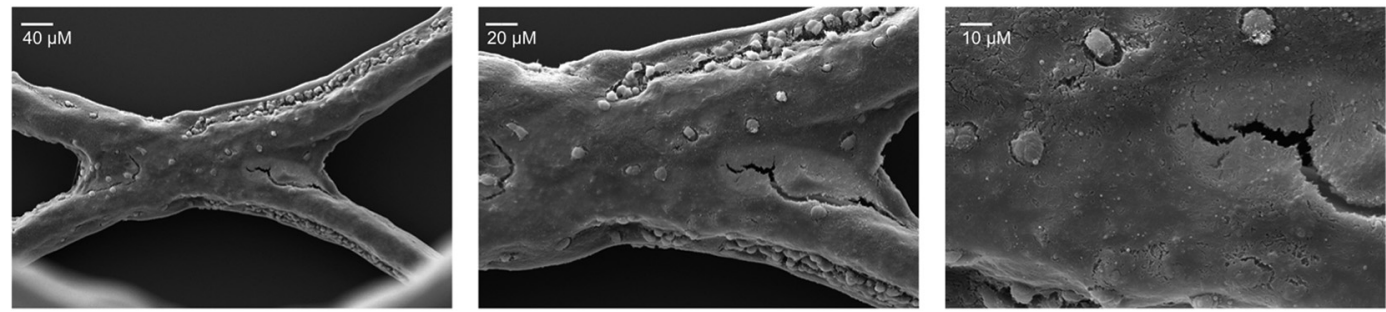

\section{Albumin coating}
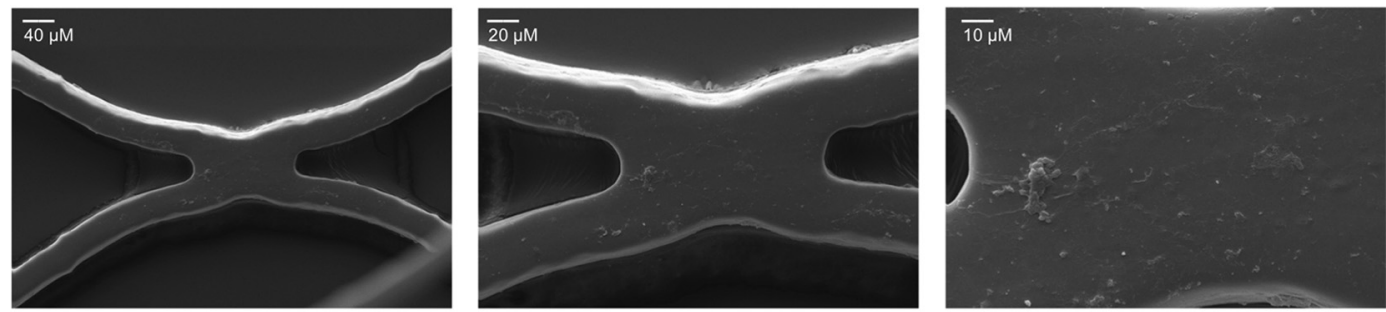

\section{Heparin coating}
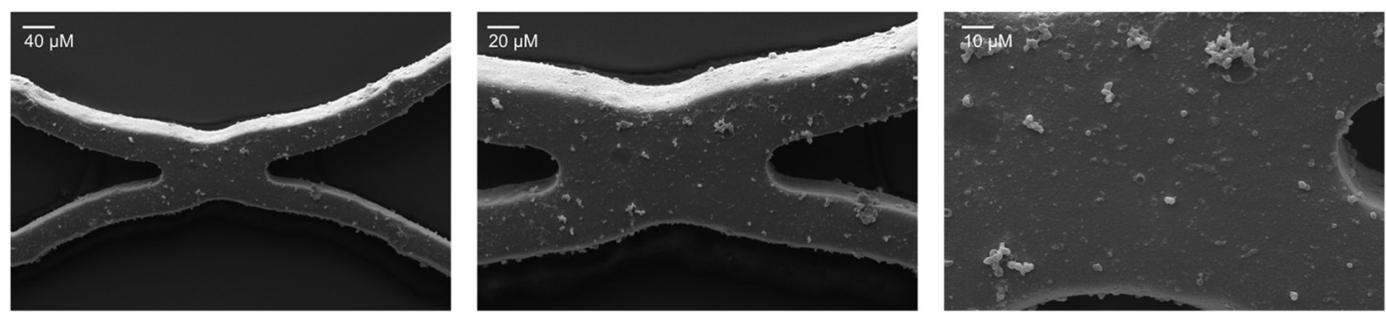

FIG 5. Thrombogenicity is reduced by albumin and heparin coating of neurovascular stents. Representative SEM images of the inner lumen of a bare metal stent or stents coated with albumin and heparin after circulation for 60 minutes at $150 \mathrm{~mL} / \mathrm{min}$ (magnification $\times 500, \times 1000$, and $\times 2500)$.

Classification of the bare metal, albumin-coated, and heparin-coated neurovascular stents based on results gained from the thrombogenicity and endothelialization models ${ }^{\mathrm{a}}$

\begin{tabular}{|c|c|c|c|c|}
\hline $\begin{array}{l}\text { Neurovascular } \\
\text { Stent }\end{array}$ & Thrombogenicity $^{\mathrm{b}}$ & $\begin{array}{c}\text { No. of } \\
\text { Experiments }\end{array}$ & Endothelialization $^{c}$ & $\begin{array}{c}\text { No. of } \\
\text { Experiments }\end{array}$ \\
\hline Bare metal & $1.87 \pm 0.4$ & 5 & $3 \pm 0$ & 3 \\
\hline Albumin-coated & $0.4 \pm 0.5$ & 5 & $2.8 \pm 0.3$ & 3 \\
\hline Heparin-coated & $0.2 \pm 0.2$ & 5 & $1.3 \pm 0.6$ & 3 \\
\hline
\end{tabular}

a Data are mean values \pm SD.

${ }^{\mathrm{b}}$ Thrombogenicity: $0=$ no thrombogenicity, $1=$ very minor thrombogenicity, $2=$ minor thrombogenicity, $3=$ moderate thrombogenicity, and $4=$ serious thrombogenicity.

c Endothelialization performance: 0 = no endothelialization, $1=$ very little endothelialization, 2 = little endothelialization, $3=$ good endothelialization, and $4=$ complete endothelialization.

system, significantly increased on circulation $(P<.001$, Fig $4 B)$. No significant differences in Sc5b-9 generation were observed between the control and the stent-containing groups.

Blood Cell Adhesion on Stents. After circulation, each stent was analyzed by using scanning electron microscopy, whereby a clear difference in thrombogenicity was observed. SEM images of each stent are exemplarily shown from 1 donor at magnifications of $\times 500, \times 1000$, and $\times 2500$ (Fig 5). The bare metal stents showed a profound adhesion of blood cells, particularly platelets, and the formation of an attenuated fibrin network for all blood donors. In contrast, no or only a few platelets were detected on the surface of the albumin- and heparin-coated stents.

Overall, the albumin- and heparin-coated stents exhibited very little thrombogenicity compared with the bare metal stents, which also did not induce strong thrombogenic reactions (Table).

\section{Endothelialization Properties of Neurovascular Stents}

Next to hemocompatibility, the rate of endothelialization is another factor in characterizing the performance of a stent. Adhesion of HUVECs on the surface of the bare metal $(n=3)$, albumin- $(n=3)$, and heparin-coated $(n=3)$ stents was thus investigated after 72 hours of dynamic incubation in a specific model. SEM images showed that the bare metal stents and the albumin-coated stents displayed good endothelialization performance. Most interesting, the adhesion of HUVECs on the surface of the heparin-coated stents was decreased compared with the bare metal and albumincoated stents (Fig 6 and Table).

\section{DISCUSSION}

The findings of our study indicate that surface coating of neurovascular stents with albumin or heparin significantly reduces platelet loss, platelet activation, and activation of the coagulation cascade. Other blood cell counts, activation of leukocytes, and the 

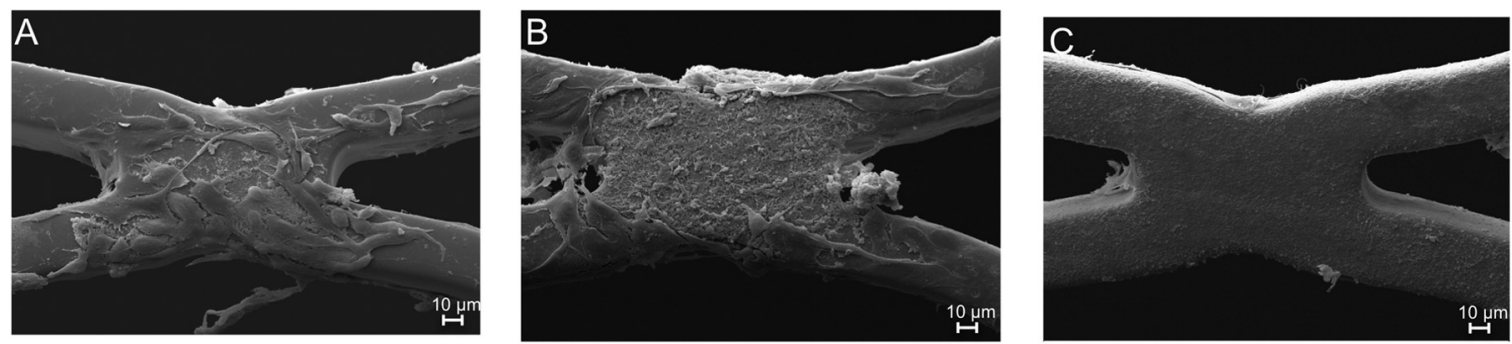

FIG 6. Endothelialization of neurovascular stents. Representative SEM images of the inner lumen of a bare metal stent $(A)$ or stents coated with albumin $(B)$ and heparin $(C)$ after incubation with human umbilical vascular endothelial cells in a rotating bioreactor for 72 hours (magnification $\times 1000)$.

complement system were not altered by the bare metal or coated stents.

To investigate the hemostatic performance of a low-profile neurovascular stent and different surface coatings, we established a dynamic in vitro model with focus on cerebral-like flow conditions and lumen size. A flow rate of $150 \mathrm{~mL} / \mathrm{min}^{-1}$ was set according to blood flow in the middle cerebral artery, resulting from measurement in 92 patients. ${ }^{13}$ A tubing diameter of $3.2 \mathrm{~mm}$ was chosen, which is an appropriate size for distal intracranial vessels like the middle cerebral artery.

In our study, platelet counts and SEM analysis showed that a high number of platelets adhere to the surface of the bare metal stents. Platelet adhesion and aggregation were seen in particular at junctions between stent struts, thus indicating the role of flow dynamic phenomena like fluid stagnation or turbulence.

Previous studies investigating biomaterials showed that heparin coating is most frequently used to increase the hemocompatibility and decrease the thrombogenicity of various biomaterials. ${ }^{14-16}$ Furthermore, albumin coating of arterial prostheses decreased activation of the coagulation cascade and platelet and leukocyte adhesion. ${ }^{17}$

In our study, both coatings showed excellent performance in preventing platelet adhesion, resulting in platelet counts similar to those in the control group without stents. Platelet activation investigated by measuring platelet granule release was also decreased by both coatings, indicating good antithrombotic surface properties.

The amount of TAT complex was also extremely reduced by the coatings; this finding shows the important role of surface material in the (intrinsic) coagulation cascade.

With regard to endothelialization, various studies have investigated the effect of heparin- or albumin-coated biomaterials in different in vitro and in vivo models. ${ }^{8,18-21}$

In our in vitro study, endothelial cell adhesion was shown on the surface of the bare metal and albumin-coated stents, whereas the heparin coating seems not to promote cell adhesion. Compared with the in vivo situation, our endothelialization experiments were performed in an isolated system containing endothelial cells and cell culture media with $2 \%$ fetal calf serum. Under physiologic conditions, the adsorption of blood plasma proteins, such as albumin and fibrinogen, occurs immediately after implantation of the biomaterial. Hence, the low amount of plasma proteins in our cell culture experiments might have influenced the endothelialization performance of the different stents; thus, further in vivo investigations are warranted.

\section{CONCLUSIONS}

Although the application of low-profile stents has greatly expanded the possibilities in treating intracranial aneurysms, improving the performance of stents with regard to biocompatibility is still necessary and can be achieved by implant surface modifications. In this in vitro study, albumin and heparin coatings reduced the thrombogenicity of the stents in comparison with the bare metal stent. Therefore, a surface that decreases platelet and coagulation activation can represent crucial progress in the treatment of intracranial aneurysms.

\section{REFERENCES}

1. Higashida RT, Smith W, Gress D, et al. Intravascular stent and endovascular coil placement for a ruptured fusiform aneurysm of the basilar artery: case report and review of the literature. J Neurosurg 1997;87:944-49

2. McLaughlin N, McArthur DL, Martin NA. Use of stent-assisted coil embolization for the treatment of wide-necked aneurysms: a systematic review. Surg Neurol Int 2013;4:43

3. O'Kelly CJ. Should stents be used in the treatment of ruptured intracranial aneurysms? J Neurosurg Sci 2011;55:27-33

4. Wanke I, Forsting M. Stents for intracranial wide-necked aneurysms: more than mechanical protection. Neuroradiology 2008;50:991-98

5. Mocco J, Fargen KM, Albuquerque FC, et al. Delayed thrombosis or stenosis following Enterprise-assisted stent-coiling: is it safe? Midterm results of the interstate collaboration of Enterprise stent coiling. Neurosurgery 2011;69:908-13, discussion 913-14

6. Ries T, Buhk JH, Kucinski T, et al. Intravenous administration of acetylsalicylic acid during endovascular treatment of cerebral aneurysms reduces the rate of thromboembolic events. Stroke 2006;37:1816-21

7. Golshani K, Ferrel A, Lessne M, et al. Stent-assisted coil embolization of ruptured intracranial aneurysms: a retrospective multicenter review. Surg Neurol Int 2012;3:84

8. Marois Y, Chakfe N, Guidoin R, et al. An albumin-coated polyester arterial graft: in vivo assessment of biocompatibility and healing characteristics. Biomaterials 1996;17:3-14

9. Mulvihill JN, Faradji A, Oberling F, et al. Surface passivation by human albumin of plasmapheresis circuits reduces platelet accumulation and thrombus formation: experimental and clinical studies. J Biomed Mater Res 1990;24:155-63

10. Chen J, Chen C, Chen Z, et al. Collagen/heparin coating on titanium surface improves the biocompatibility of titanium applied as a blood-contacting biomaterial. J Biomed Mater Res A 2010;95:341-49

11. Johnson I, Perchy D, Liu H. In vitro evaluation of the surface effects on magnesium-yttrium alloy degradation and mesenchymal stem cell adhesion. J Biomed Mater Res A 2012;100:477-85 
12. Avci-Adali M, Kobba J, Neumann B, et al. Application of a rotating bioreactor consisting of low-cost and ready-to-use medical disposables for in vitro evaluation of the endothelialization efficiency of small-caliber vascular prostheses. J Biomed Mater Res B Appl Biomater 2013;101:1061-68

13. Zhao M, Amin-Hanjani S, Ruland S, et al. Regional cerebral blood flow using quantitative MR angiography. AJNR Am J Neuroradiol 2007;28:1470-73

14. Hirsh J, Siragusa S, Cosmi B, et al. Low-molecular-weight heparins (LMWH) in the treatment of patients with acute venous thromboembolism. Thromb Haemost 1995;74:360-63

15. Blezer R, Fouache B, Willems GM, et al. Activation of blood coagulation at heparin-coated surfaces. J Biomed Mater Res 1997;37:108-13

16. Klement P, Du YJ, Berry L, et al. Blood-compatible biomaterials by surface coating with a novel antithrombin-heparin covalent complex. Biomaterials 2002;23:527-35

17. Kottke-Marchant K, Anderson JM, Umemura Y, et al. Effect of albu- min coating on the in vitro blood compatibility of Dacron arterial prostheses. Biomaterials 1989;10:147-55

18. Bae IH, Park IK, Park DS, et al. Thromboresistant and endothelialization effects of dopamine-mediated heparin coating on a stent material surface. J Mater Sci Mater Med 2012;23:1259-69

19. Moer R, Myreng Y, Mølstad P, et al. Stenting in small coronary arteries (SISCA) trial: a randomized comparison between balloon angioplasty and the heparin-coated beStent. J Am Coll Cardiol 2001;38:1598-603

20. Serruys PW, Emanuelsson H, van der Giessen W, et al. Heparincoated Palmaz-Schatz stents in human coronary arteries: early outcome of the Benestent-II Pilot Study. Circulation 1996; 93:412-22

21. Blindt R, Vogt F, Astafieva I, et al. A novel drug-eluting stent coated with an integrin-binding cyclic Arg-Gly-Asp peptide inhibits neointimal hyperplasia by recruiting endothelial progenitor cells. J Am Coll Cardiol 2006;47:1786-95 